\title{
COMPUTATION OF THE DYNAMIC APERTURE OF 2D GENERIC MAPS USING INVARIANT MANIFOLDS
}

\author{
Massimo Giovannozzi*, INFN Sezione di Bologna, ITALY
}

\begin{abstract}
In this paper the phase-space of generic $2 \mathrm{D}$ area-preserving polynomial mappings is studied. These mappings modelize the transverse dynamics of a flat beam in a circular machine dominated by nonlinear magnetic errors. In particular, the problem of computing the dynamic aperture, i.e. the region in phase-space where stable motion occurs, is considered. The main result is that the boundary of the stability domain is given by the invariant manifolds emanating from the outermost unstable fixed point of low period (one or two). This study extends previous results obtained for reversible areapreserving polynomial maps of the plane.
\end{abstract}

\section{INTRODUCTION}

One of the main topics in the study of nonlinear Hamiltonian systems is the determination of the region in phasespace where bounded motion occurs (also called stability domain or dynamic aperture). For two-dimensional nonlinear systems, it is possible to define unambiguously an area in phase-space where the motion is stable for arbitrarily long periods. Around the origin, which is usually chosen to be a stable fixed point, there are closed curves (1D KAM tori), and wherever the nonlinear frequency satisfies a resonant condition, the invariant curves are broken into islands. When nonlinearities are dominant, one reaches a stability border beyond which a fast escape to infinity occurs. This stability border is what we have called the dynamic aperture. In this picture the KAM tori separate different phase-space domains: therefore, there exists a last connected invariant curve whose interior represents a set of stable initial conditions. Outside this curve, there can only be islands of stability, scattered in the sea of initial conditions which escape to infinity.

The evaluation of the stability domain is not only an important issue from a theoretical point of view, but also a key problem in many applications. For instance, the size of the stability domain is the main source of concern in the design of a circular particle accelerator. This parameter imposes tight constraints on the magnetic lattice of the machine and a good insight into the sources of instabilities is necessary to ensure good performance.

Our approach to the problem of determining the stability domain of a $2 \mathrm{D}$ polynomial map, which generalizes the results of [1-3], consists in computing the invariant manifolds of the outermost hyperbolic fixed point. Thanks to

\footnotetext{
* Present address: CERN PS Division
}

the homoclinic and/or heteroclinic intersections, the invariant manifolds related with different fixed points are connected with each other generating the homoclinic tangle. This structure is shown to be the border of the stability domain. A result obtained by Friedland and Milnor [4], allows the proof of the existence of hyperbolic fixed points of low period (one or two) for generic area-preserving polynomial maps of the plane, thus showing that our method is generically applicable to determine the stability domain.

\section{POLYNOMIAL MAPS AND FIXED POINTS}

\subsection{A classification Theorem}

In 1969 [5], Hénon showed that every quadratic mapping of the plane can be reduced to the simple form

$$
(x, y) \stackrel{\mathbf{h}_{2}}{\longmapsto}\left(y, y^{2}+c-\delta x\right),
$$

where the parameter $\delta$ represents the constant jacobian. Friedland and Milnor [4] found a way to generalize the result obtained by Hénon to arbitrary degree polynomial maps. The result of their studies can be summarized as follows

THEOREM 1 Every polynomial map can be written as the composition of

$$
\left(x_{0}, x_{1}\right) \stackrel{\mathbf{h}_{d_{1}}}{\longmapsto}\left(x_{1}, x_{2}\right) \stackrel{\mathbf{h}_{d_{2}}}{\longmapsto} \ldots \stackrel{\mathbf{h}_{d_{m}}}{\longmapsto}\left(x_{m}, x_{m+1}\right),
$$

where $\mathbf{h}_{d_{i}}$ is a generalized Hénon map, namely

$$
\mathbf{h}_{d_{i}}(x, y)=\left(y, p_{i}(y)-\delta_{i} x\right)
$$

and $p_{i}(y)$ is a polynomial of degree $d_{i} \geq 2$. This composition can be chosen so that the leading coefficient, i.e. the coefficient of the highest degree, in each polynomial $p_{i}$ is \pm 1 , and so that the next highest coefficient is zero. The resulting normal form is unique up to a finite number of choices.

Thus every polynomial transformation is defined inductively by $x_{i+1}=p_{i}\left(x_{i}\right)-\delta_{i} x_{i-1}$, with

$$
p_{i}\left(x_{i}\right)= \pm x_{i}^{d_{i}}+\left(\text { terms of degree } \leq d_{i}-2\right) .
$$

It is readily seen that this normal form depends on exactly $d_{1}+\cdots d_{m}$ parameters. 


\subsection{Fixed points of area-preserving maps}

A fixed point of a polynomial map $\mathbf{g}$ is a root of the equation

$$
\mathbf{g}(x, y)=(x, y)
$$

The solutions of Eq. (5) can be classified by considering the trace of the linearization $\mathbf{g}_{L}$ around the fixed point. In the area-preserving case, the situation is as follows

$$
\left|\operatorname{Tr}\left(\mathbf{g}_{\mathrm{L}}\right)\right| \begin{cases}<2 & \text { the fixed point is elliptic } \\ =2 & \text { the fixed point is parabolic } \\ >2 & \text { the fixed point is hyperbolic }\end{cases}
$$

In the first case the fixed point is stable while in the third case it is unstable. The following result can be proved:

THEOREM 2 Given an area-preserving polynomial map g of degree $d$ then

- the outermost fixed point is always hyperbolic if $d$ is even or $d$ is odd and the leading term is positive;

- the outermost fixed point of $\mathbf{g}^{2}$ is always hyperbolic if $d$ is odd and the leading term is negative.

\section{INVARIANT MANIFOLDS}

For a hyperbolic fixed point $\mathbf{x}_{\mathrm{hyp}}$, the eigenvectors of the linearization of the map $\mathbf{g}_{\mathrm{L}}$ around $\mathbf{x}_{\mathrm{hyp}}$ define two linear sets in the plane, along which the motion induced by the linearized map has an expanding or a contracting behaviour.

We can extend these sets to the original nonlinear map $\mathbf{g}$, i.e. it is possible to define two manifolds emanating from the unstable fixed point, called $\mathcal{W}^{\mathrm{u}}\left(\mathbf{x}_{\text {hyp }}\right)$ and $\mathcal{W}^{\mathrm{s}}\left(\mathbf{x}_{\text {hyp }}\right)$, having the same expanding (superscript $\mathrm{u}$ ) or contracting (superscript s) behaviour. The eigenvectors of $\mathbf{g}_{\mathrm{L}}$ are tangential to $\mathcal{W}^{\mathrm{u}, \mathrm{s}}\left(\mathbf{x}_{\mathrm{hyp}}\right)$ at the fixed point.

The invariant manifolds have at least the hyperbolic fixed point as intersection. An additional intersection, $\mathbf{x}_{\mathrm{hom}}$, is either called homoclinic or heteroclinic depending on whether the two intersecting manifolds emanate from the same hyperbolic fixed point. Provided the two manifolds are non-tangential at the point $\mathbf{x}_{\mathrm{hom}}$, it can be proven that the set of intersections is countable. Therefore, unless the two manifolds coincide completely, which occurs in the integrable case, they will oscillate around each other.

Due to the area-preserving character of the map, the area enclosed between two successive intersections remains constant. As the period of the motion tends to infinity approaching the hyperbolic fixed point, the distance between successive intersections decreases exponentially, leading to larger and larger oscillations close to the hyperbolic fixed point.

Efficient algorithms have been developed [6] to construct the whole sets $\mathcal{W}^{\mathrm{u}, \mathrm{s}}\left(\mathbf{x}_{\text {hyp }}\right)$. They allow the reconstruction of the invariant manifolds with a uniform accuracy using a relatively small number of initial conditions together with efficient interpolation schemes.
For the purpose of this study, a simpler approach has been implemented. In fact, it turns out, that it is sufficient to iterate many times a set of initial conditions belonging to a small part of these manifolds in the vicinity of $\mathbf{x}_{\mathrm{hyp}}$. Moreover, these initial conditions can be chosen on the eigenvalues of the linearized map provided their distance to the hyperbolic fixed point is sufficiently small.

\section{STABILITY DOMAIN}

\subsection{Analytical results}

The stability domain $\mathcal{K}$ of a polynomial map $\mathbf{g}$ is defined as the region in phase-space where stable motion occurs. An initial condition $\left(x_{0}, y_{0}\right)$ is stable if the sequence of iterates $\left(x_{n}, y_{n}\right)=\mathbf{g}^{n}\left(x_{0}, y_{0}\right)$ is bounded for both positive and negative $n$. One can prove [4] that $\mathcal{K}$ is compact and of positive Lebesgue measure.

Consider the following set

$$
\partial \mathcal{K}=\mathcal{W}^{\mathrm{s}}\left(\mathbf{y}_{\text {hyp }}\right) \bigcup \mathcal{W}^{\mathrm{u}}\left(\mathbf{y}_{\text {hyp }}\right),
$$

where $\mathbf{y}_{\text {hyp }}$ is the outermost hyperbolic fixed point. In case the degree of $\mathbf{g}$ is odd and the coefficient of the leading term is negative, the hyperbolic fixed point used in Eq. (7) refers to the second iterate of the map $\mathbf{g}^{2}$, while in the other cases it represents the fixed point of $\mathbf{g}$. It is readily seen that $\partial \mathcal{K}$ is invariant under the dynamics generated by $\mathbf{g}$, hence, an orbit cannot cross this set. The geometrical structure of $\partial \mathcal{K}$ is extremely complex. In fact, the invariant manifolds emanating from different hyperbolic fixed points of the map or its second iterate, present homoclinic and, possibly, heteroclinic intersections. This ensures that $\mathcal{K}$ encloses a finite region of phase-space. Furthermore, the manifolds emanating from hyperbolic fixed points of higher period, i.e. solutions of

$$
\mathbf{g}^{m}(x, y)=(x, y)
$$

might also have intersections with $\partial \mathcal{K}$ : the result is a dense network of manifolds emanating from the outermost hyperbolic fixed point, with an infinite number of intersections. The dynamics outside $\mathcal{K}$ is trivial: no bounded motion can occur and the same happens for points trapped inside the homoclinic tangle.

Finally, points belonging to the region inside $\partial \mathcal{K}$ are stable, as they cannot leave this region without crossing $\partial \mathcal{K}$, and violating the invariance property.

\subsection{Numerical results}

The result presented in this paper have been obtained with the program GIOTTO [7]. GIOTTO allows the study of the dynamics of 2D systems using numerical tools (such as frequency analysis, evaluation of Lyapunov exponents, visualization of phase-space portrait, computation of fixed point computation and evaluation of homoclinic tangle) as well as analytical tools (such as normal forms).

The first model analyzed consists of a polynomial map 
of degree six

$$
\mathbf{h}_{6}=\mathbf{h}_{2} \circ \mathbf{h}_{3}
$$

where

$$
\mathbf{h}_{3}=\left(y, y^{3}+\alpha y+\beta-x\right) \quad \mathbf{h}_{2}=\left(y, y^{2}+\gamma-x\right) .
$$

The parameters have been set equal to the following values $\alpha=-0.856, \beta=-0.164, \gamma=-0.120$. In Fig. 1, the key

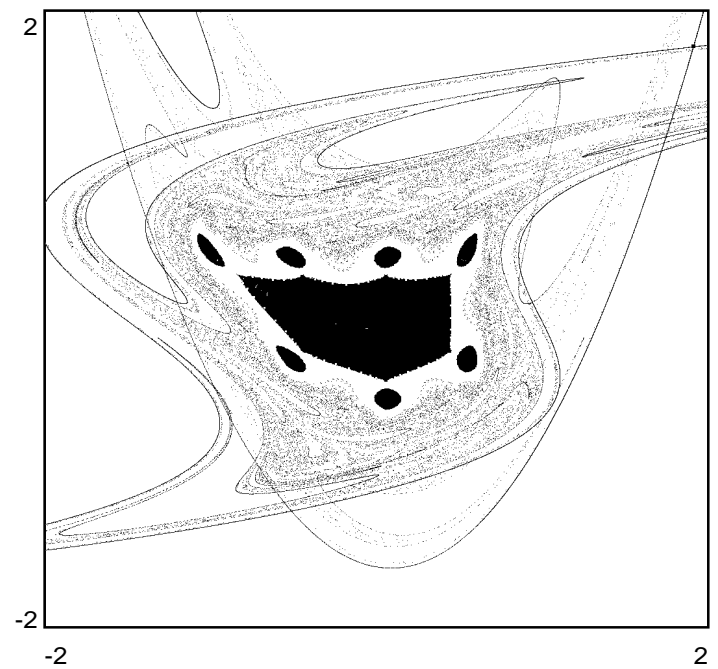

Figure 1: Stability domain (black area) and the invariant manifolds emanating from the unstable fixed point of period one of $\mathbf{h}_{6}$.

result is shown (the black area represents the set $\mathcal{K}$ ). This has been computed by simply iterating the map $\mathbf{h}_{6}$ over a rectangular grid of initial conditions and plotting the initial conditions which stay bounded after 10000 turns. On top of the set $\mathcal{K}, \partial \mathcal{K}$ is superimposed. It is apparent that $\partial \mathcal{K}$ bounds $\mathcal{K}$.

The next step would be to consider an odd degree map. The chosen models are:

$$
\mathbf{h}_{9 \pm}=\mathbf{h}_{3 \pm}^{\prime} \circ \mathbf{h}_{3}
$$

where

$\mathbf{h}_{3 \pm}^{\prime}=\left(y, \pm y^{3}+\alpha y+\beta-x\right) \quad \mathbf{h}_{3}=\left(y, y^{3}+\gamma y+\delta-x\right)$.

The parameters have been set equal to $\alpha=-0.712$, $\beta=-0.272, \gamma=0.268, \delta=0.680$ for $\mathbf{h}_{9+}$, while $\alpha=-0.172, \beta=0.352, \gamma=0.916, \delta=0.348$ for $\mathbf{h}_{9-}$ has been chosen. In Fig. 2 the set $\mathcal{K}$ is depicted together with $\partial \mathcal{K}$. The outermost fixed point is hyperbolic and it can be used to construct the invariant manifolds and the set $\partial \mathcal{K}$. Finally, in Fig. 3 the two sets $\mathcal{K}$ and $\partial \mathcal{K}$ (relative to the fixed point of period two) are shown. It is clearly seen that the homoclinic tangle generated by either the unstable fixed point of period one or the unstable fixed point of period two represents the border of the region of stable motion for $\mathbf{h}_{9+}, \mathbf{h}_{9-}$. Although the phase-space topology of the three models presented here is completely different, nevertheless the information on the hyperbolic fixed points

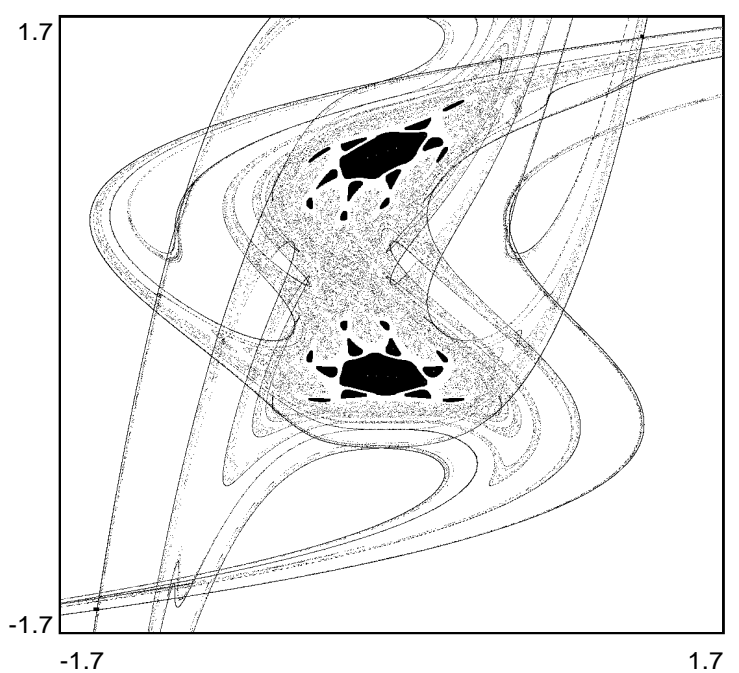

Figure 2: Stability domain (black area) and invariant manifolds emanating from the outermost unstable fixed point of period one for $\mathbf{h}_{9+}$.

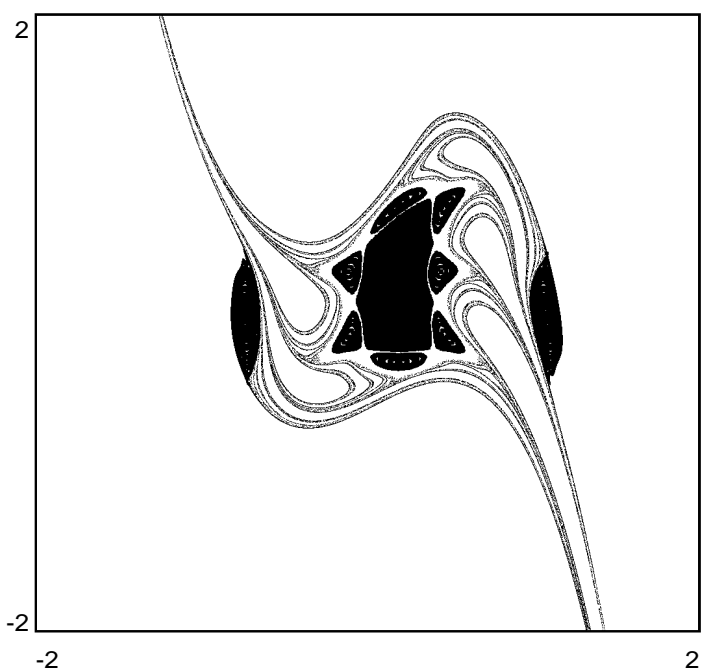

Figure 3: Stability domain (black area) and invariant manifolds from the outermost unstable fixed point of period two for $\mathbf{h}_{9-}$.

of low period is sufficient to reconstruct the border of the stability domain, thanks to the phenomenon of homoclinic and heteroclinic intersections.

\section{REFERENCES}

[1] M. Giovannozzi, Phys. Lett. A182 (1993) 255.

[2] A. Bazzani, M. Giovannozzi, G. Servizi, E. Todesco and G. Turchetti, Physica D64 (1993) 66.

[3] M. Giovannozzi, Phys. Rev. E 53 (1996) 6403.

[4] S. Friedland and J. Milnor, Ergod. Th. \& Dynam. Sys. 9 (1989) 67.

[5] M. Hénon, Q. Appl. Math. 27 (1969) 291.

[6] D. Hobson, J. of Comp. Phys. 104 (1993) 14.

[7] D. Bortolotti, M. Giovannozzi, G. Servizi, E. Todesco and M. N. Vrahatis, Int. J. Mod. Phys. C6 (1995) 651. 